\title{
X-Ray Diffraction Computed Nanotomography Applied to Solve the Structure of Hierarchically Phase-Separated Metallic Glass
}

Mihai Stoica ${ }^{1}$, Baran Sarac ${ }^{2}$, Florian Spieckermann ${ }^{3}$, Jonathan Wright ${ }^{4}$, Christoph Gammer $^{2}$, Junhee Han ${ }^{5}$, Petre F. Gostin ${ }^{6}$, Jürgen Eckert ${ }^{2,3}$, Jörg F. Löffler ${ }^{1}$

${ }^{1}$ Laboratory of Metal Physics and Technology, Department of Materials, ETH Zurich, 8093 Zurich, Switzerland

2 Erich Schmid Institute of Materials Science, Austrian Academy of Sciences (ÖAW), 8700 Leoben, Austria

${ }^{3}$ Chair of Materials Physics, Department of Materials Science, Montanuniversität Leoben, 8700 Leoben, Austria

${ }^{4}$ European Synchrotron Radiation Facility (ESRF), 38042 Grenoble, France

${ }^{5}$ Korea Institute for Rare Metals (KIRAM), Korea Institute of Industrial Technology (KITECH), Yeonsu-Gu, 21999 Incheon, South Korea

6 School of Metallurgy and Materials, University of Birmingham, Edgbaston, Birmingham B15 2TT, United Kingdom

Fig. S1 displays the experimental set-up schematically, with a real picture of the measured sample. The scan on the $Y$ axis extended over $100 \mu \mathrm{m}$, with each line scan consisting of 200 points equally spaced at $0.5 \mu \mathrm{m}$. The scan on $\omega$, i.e. the rotational scan, was performed at every $1^{\circ}$ for a total of $180^{\circ}$, meaning that the total number of XRD patterns was $N_{Y} \times N_{\omega}=36,000$. The voxel size is the same as the step size in the scan, i.e. $500 \mathrm{~nm} \times 500 \mathrm{~nm} \times 500 \mathrm{~nm}$.

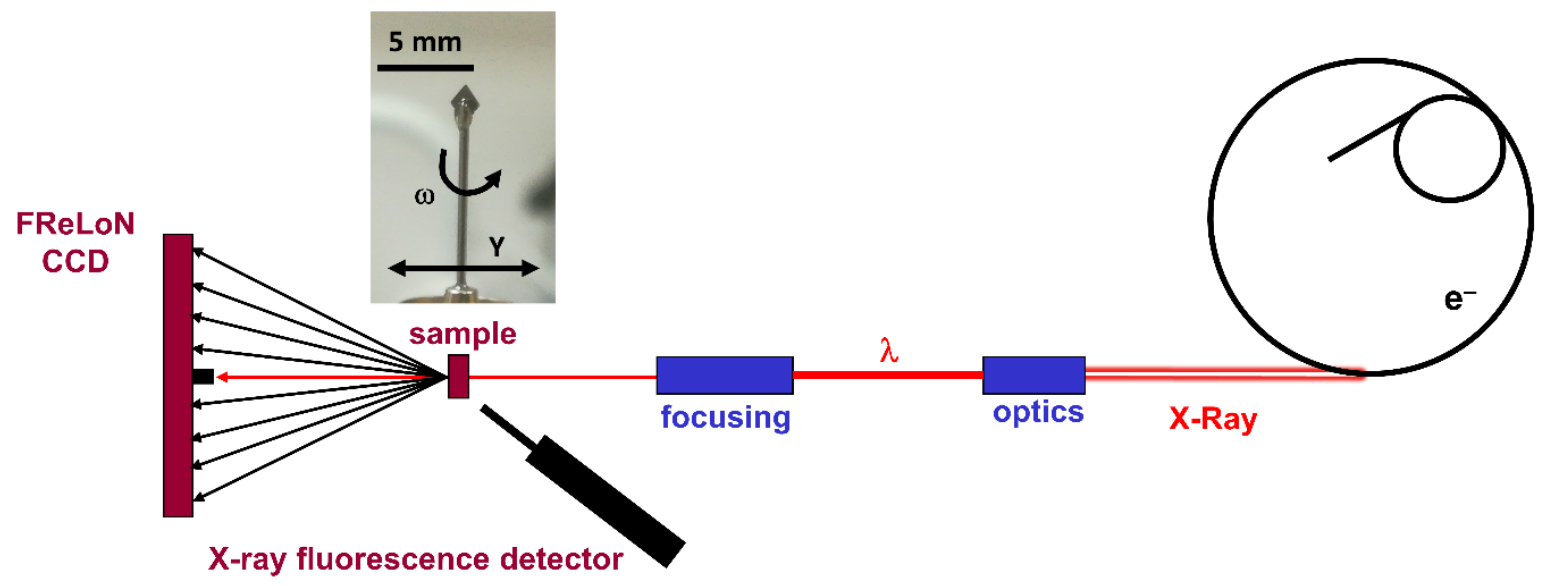

Fig. S1. Schematic drawing of the experimental set-up for XRD-nCT. The inset photo illustrates the sample mounting, with the small ribbon fragment located at the top of the vertical pin. 
Fig. S2 displays schematically the sample in the beam, detailing the $X, Y, Z$ and $\omega$ axes. As it is customary, the $X$ direction is along the beam path, while $Y$ and $Z$ are perpendicular to it. The scan procedure was repeated for three different slices, Z1, Z2 and $Z 3$, equally distanced at $500 \mathrm{~nm}$. The beam is illustrated here as having the same thickness as the illuminated slice.

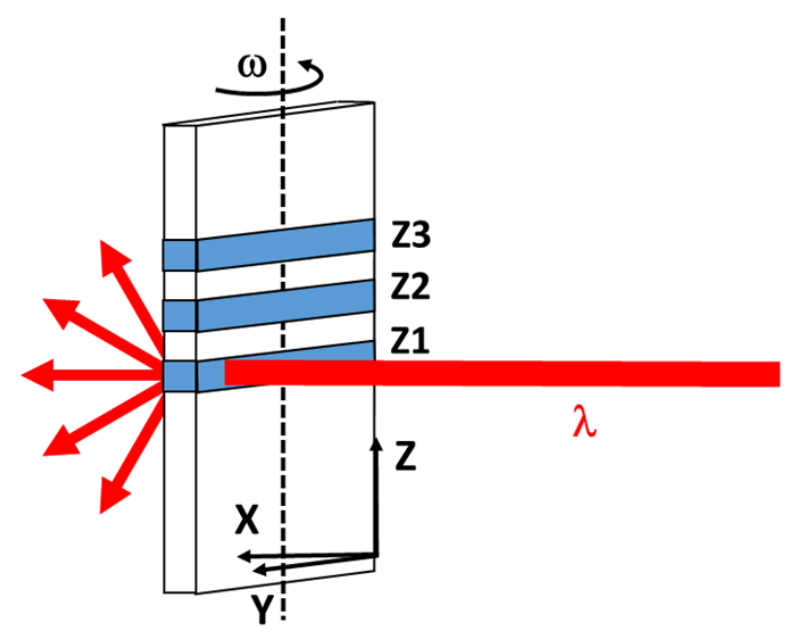

Fig. S2. Schematic drawing of the sample in the beam, showing the scanned sections; the three orthogonal directions $X$ (along the beam path), $Y$ (perpendicular to the beam path, forming with $X$ the horizontal plane) and $Z$ (perpendicular to the beam path, forming with $X$ the vertical plane); and the rotation axis $\omega$.

The quality of the reconstructed axial slice depends on the total number of projections, which should satisfy the Nyquist-Shannon sampling theorem: ${ }^{1,2}$

$$
N_{\text {images }}=N_{\mathrm{Y}} N_{\omega} \geq \frac{\pi}{2} \text { 2D_image_size_in_pixels }
$$

The translational step on the $Y$ axis is usually chosen in dependence of the beam size. In this case the minimum number of equally spaced angular rotation steps is calculated according to Eq. 1. In our case, taking into account that the beam is $500 \mathrm{~nm}$ wide, the minimum scanning interval is the beam itself. The scan on $\omega$ at every degree was chosen to increase the quality of the reconstruction. Then, at least theoretically, with the current number of data sets it results that 2D_image_size_in_pixels $\leq \frac{2}{\pi} N_{\mathrm{Y}} N_{\omega}=$ 22,918 pixels. Physically, the reconstructed image is $100 \mu \mathrm{m} \times 100 \mu \mathrm{m}$ large, therefore 1 pixel should correspond to an area of $0.436 \mu \mathrm{m}^{2}$. Accordingly, the $2 \mathrm{D}$ resolution of the reconstructed image would be $660 \mathrm{~nm}$.

However, as it is often mentioned in literature, ${ }^{2,3}$ "Undersampling remains one of the worst sins one can commit in this field. You should never claim any resolution level beyond 2/3rd of the Nyquist frequency". ${ }^{3}$ Also, other unknown drift or noise problems may affect the real resolution of the reconstructed image. At two-thirds of the Nyquist frequency the pixel is $0.654 \mu \mathrm{m}^{2}$ and the $2 \mathrm{D}$ resolution becomes $809 \mathrm{~nm}$. If one considers only half of the pixels in the Nyquist-Shannon sampling theorem, then the pixel has an area of $0.872 \mu \mathrm{m}^{2}$ and the resolution becomes $933 \mathrm{~nm}$. 


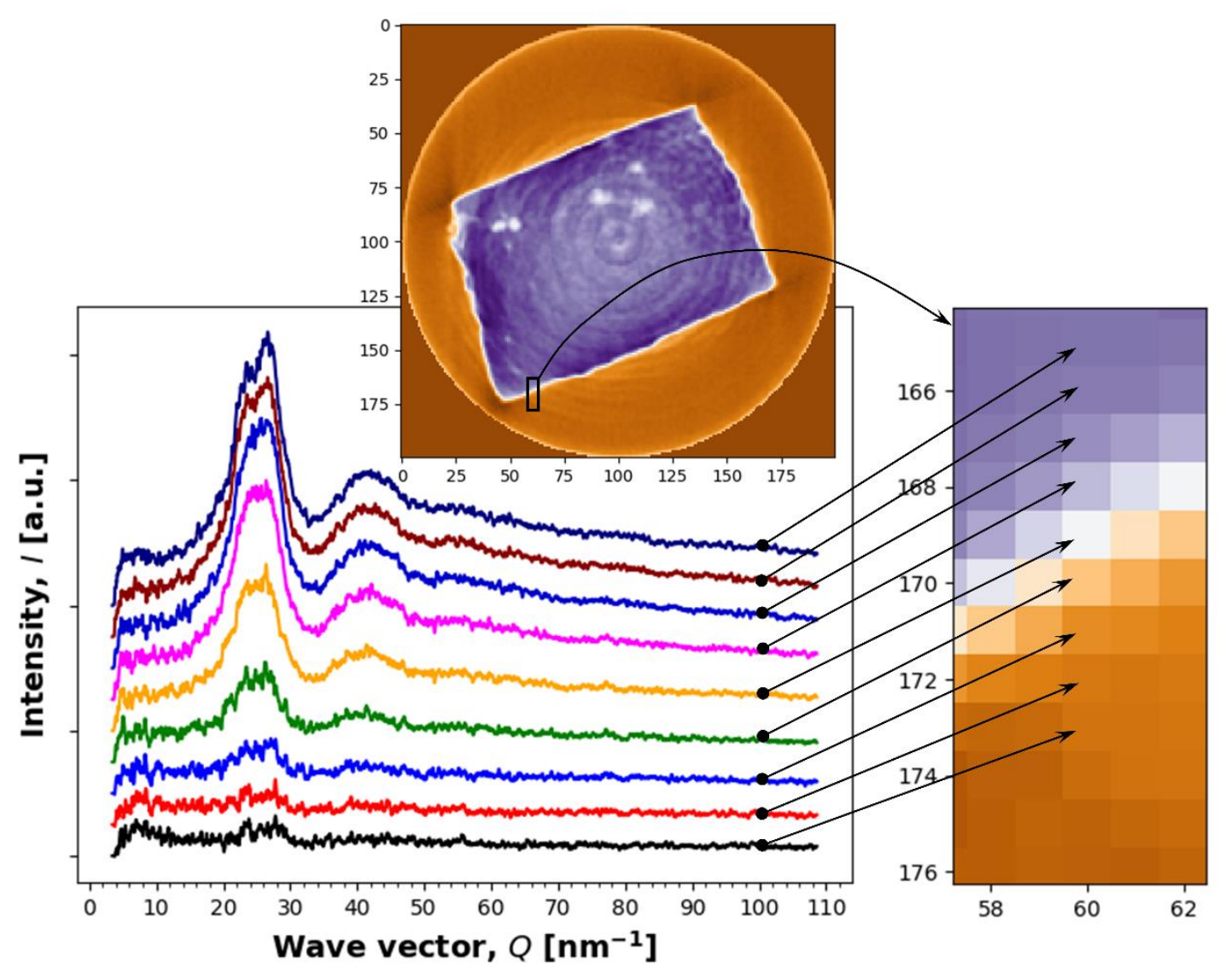

Fig. S3. Reconstructed XRD patterns taken for every pixel across a margin of the sample. The upper picture depicts the tomographic reconstruction, plotted in a pixels $\times$ pixels system of coordinates. The investigated margin is marked by a black rectangle and expanded on the right.

To further analyze the resolution, Fig. S3 shows the reconstructed XRD patterns for adjacent pixels across a margin of the sample. The upper picture depicts the tomographic reconstruction, which for the purpose of illustration is plotted in a pixels $\times$ pixels system of coordinates. The investigated margin is marked by a black rectangle and expanded on the right of the figure. The first pattern presented in Fig. S3 corresponds to a $(60,173)$ pixel and the last one to a $(60,165)$ pixel. There it is a sharp difference between the $(60,171),(60,170)$ and $(60,169)$ pixels, proving that differences from one pixel to the next can be clearly detected. Hence, it is reasonable to assume that our reconstructed image has a sub-micron $2 \mathrm{D}$ resolution.

\section{References}

1. Cersoy, S.; Leynaud, O.; Álvarez-Murga, M.; Martinetto, P.; Bordet, P.; Boudet, N.; Chalmin, E.; Castets, G.; Hodeau, J.L. Laboratory Implementation of X-Ray Diffraction/Scattering Computed Tomography. J. Appl. Cryst. 2015, 8, 159-165.

2. Hsieh, J. Computed Tomography: Principles, Design, Artifacts, and Recent Advances, SPIE Press and Wiley Interscience, Bellingham, 2009.

3. Image Science, Advanced Image Processing \& Image Analysis. www.imagescience.de/fsc (January 15, 2021). 